\title{
Unequal burden of mortality from gastric cancer in Brazil and its regions, 2000-2015
}

\author{
Lucia Libanez Bessa Campelo Braga ${ }^{1}$. Alberto Novaes Ramos $\mathrm{Jr}^{2} \cdot$ Manuel Bonfim Braga Neto ${ }^{1}$. \\ Anderson Fuentes Ferreira ${ }^{2}$. Dulciene Maria Magalhães Queiroz ${ }^{3}$. Danielle Calheiros Campelo Maia ${ }^{1}$. \\ Carlos Henrique Alencar ${ }^{2}$. Jorg Heukelbach ${ }^{2,4}$
}

Received: 7 June 2018 / Accepted: 14 December 2018 / Published online: 2 January 2019

(c) The International Gastric Cancer Association and The Japanese Gastric Cancer Association 2019

\begin{abstract}
Background Gastric cancer (GC) is an important cause of morbidity and mortality worldwide. However, population-based data on GC mortality dynamics in low and middle income countries are scarce.

Methods We analyzed GC mortality in Brazil based on all GC-related deaths registered 2000-2015.

Results A total of 17,374,134 deaths were recorded, with GC identified in 214,808 (1.24\%) cases-203,941 (94.9\%) as underlying cause, and 10,867 (5.1\%) as associated cause of death. Adjusted rates for age and sex was 6.85 deaths/100,000 inhabitants [95\% confidence interval (CI) 6.73-6.97]. The highest mortality rates were found in males [10.00; rate ratio (RR) $1.85 ; 95 \%$ CI $1.78-1.91 ; p<0.0001]$ and patients $\geq 45$ years of age $(24.98$; RR $3.79 ; 95 \%$ CI $3.55-4.05 ; p<0.0001)$. The South (7.56; RR 1.62; 95\% CI 1.50-1.76; $p<0.0001)$ and Southeast $(7.36$; RR 1.59; 95\% CI 1.48-1.71; $p<0.0001)$ regions had the highest regional rates. Spatial and spatiotemporal high-risk mortality areas in 2004-2007 were located mainly in the South, Southeast, and Central-West regions. After 2008, the Northeast region became a high-risk area, especially Ceará State. Conclusion GC remains a significant public health problem with high mortality burden and unequal distribution in Brazilian states. The new patterns in poorer regions and the high risk in some specific populations show a clear process of epidemiological transition over time. There is a need to strengthen nationwide epidemiological monitoring, surveillance, prevention, and control for GC in the country.
\end{abstract}

Keywords Gastric cancer · Mortality $\cdot$ Spatial analysis $\cdot$ Brazil

Lucia Libanez Bessa Campelo Braga

lucialib@terra.com.br

1 Clinical Research Unit/Biomedicine Institute, Department of Internal Medicine, School of Medicine, Federal University, Rua Coronel Nunes de Melo 1315, Rodolfo Teófilo, Fortaleza, Ceara 60430270, Brazil

2 Department of Community Health, School of Medicine, Federal University of Ceará, Fortaleza, Brazil

3 Laboratory of Research in Bacteriology, School of Medicine, Federal University of Minas Gerais, Belo Horizonte, Brazil

4 Division of Tropical Health and Medicine, College of Public Health, Medical and Veterinary Sciences, James Cook University, Townsville, Australia

\section{Introduction}

Gastric cancer (GC) is the fifth most common malignant tumor worldwide and the third cause of cancer-related deaths and disability-adjusted life years (DALYs) [1].

GC is associated with high morbidity and mortality burden, especially in low and middle income countries [6]. The highest incidences of GC have been observed in East Asia, Eastern Europe, and South America, whereas the lowest rates occur in North America, South Asia, North and East Africa, and Australia [1, 2]. These geographic differences are thought to be caused by differing risk factors, such as dietary habits, smoking, H. pylori infection, genetic factors, and socioeconomic development [2-5].

In the past decades, mortality rates have been decreasing in most countries, likely due to declining prevalence of H. pylori infection and smoking rates, and improved early detection and treatment $[2,6]$. Five-year survival rates are 
below $30 \%$ in most countries. However, the rates reach approximately $50 \%$ in South Korea and Japan, largely attributed to comprehensive screening programs, aimed at early detection [7].

In Brazil, GC is the fourth most common cancer among men and the fifth most common among women [8]. In more than $75 \%$ of cases, GC is diagnosed in advanced stages [8, 9]. Five-year survival rates in Brazil declined in the past decades, in contrast to the upward trend seen in most other countries $[1,2,6,9]$.

Brazil is a country with continental dimensions and considerable social and economic disparities between regions. Low socioeconomic status has been associated with higher risk of GC incidence, regardless of sex or country of origin [10]. There are few studies on GC-related mortality showing different epidemiological patterns among the different Brazilian geographic regions $[9,11,12]$. Overall, male sex, increased age and lower socio-economic status are associated with higher mortality $[9,10]$. The mortality rates in the north and northeast, Brazil's less developed regions, are expected to increase $[9,11]$. However, these studies considered only underlying causes of death, and there are no systematic nationwide studies evaluating the spatiotemporal patterns of GC mortality rates. In this study, we analyzed the spatial and spatiotemporal high-risk clusters of GC-related mortality over a period of 16 years.

\section{Methods}

\section{Study setting}

Brazil is South America's largest country, with an estimated population of about 205 million of inhabitants in 2015. The country has the highest gross domestic product (GDP_US\$ 1800 billion) of Latin American Countries and the 9th in the world (International Monetary Fund World Economic Outlook, October-2015).

The country is divided into five geographic and administrative regions (South, Southeast, Central-West, North, and Northeast), which are grouped into 27 Federal Units (1 Federal District, 26 States) for purposes of statistical interpretations, management of public functions and guiding the application of public policies (Fig. 1).

There are large disparities between the richest and poorest population strata (Gini index-labour income-of 0.549 in 2017), and there is a wide demographic, cultural and socioeconomic diversity between Brazilian regions, with Gini
Fig. 1 Brazil with its regions and 27 federal units

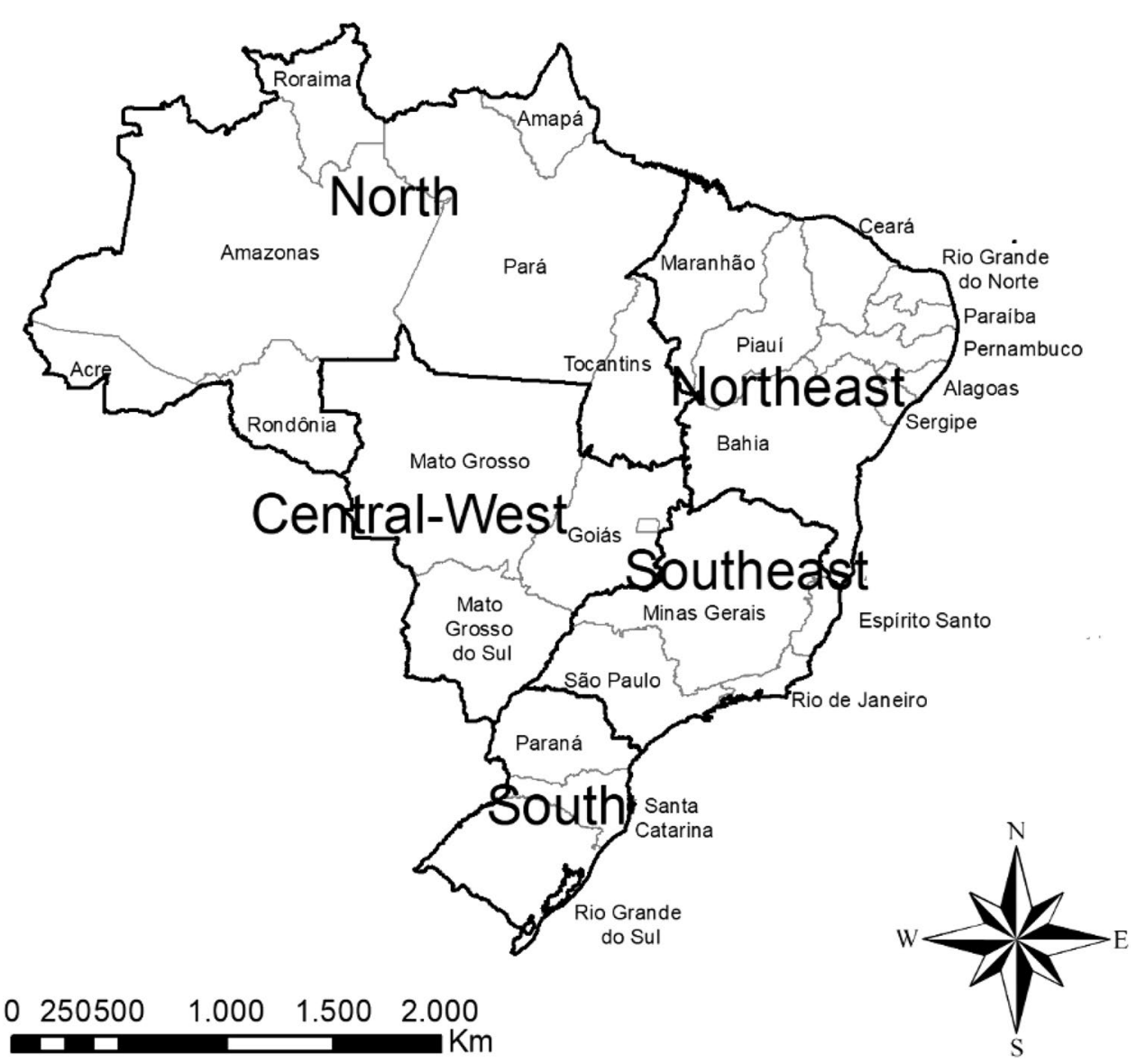


indexes ranging from 0.477 in the South to 0.567 in the Northeast region in 20.

\section{Study population}

We included all registered deaths that occurred in Brazil from 2000 to 2015 and in which GC was recorded on death certificates, either as underlying or as associated causes of death. Underlying cause of death was defined as GC being the disease that initiated the sequence of clinical events leading directly to death. The Brazilian death certificate follows the standard WHO form, with only a single underlying cause. GC as associated cause of death was considered, if mentioned as immediate, intermediate, or contributing cause. We used a classical approach of analysis of multiple causes of death, including all deaths related to GC as underlying and/or associated causes.

GC was defined based on the Tenth Revision of the International Statistical Classification of Diseases and Related Health Problems (ICD-10). We included all deaths in the group code $\mathrm{C} 16$ (Malignant neoplasm of stomach): C16.0 Malignant neoplasm: Cardia; C16.1 Malignant neoplasm: Fundus of stomach; C16.2 Malignant neoplasm: Body of stomach; C16.3 Malignant neoplasm: Pyloric antrum; C16.4 Malignant neoplasm: Pylorus, C16.5 Malignant neoplasm: Lesser curvature of stomach, unspecified; C16.6 Malignant neoplasm: Greater curvature of stomach, unspecified; C16.8 Malignant neoplasm: Overlapping lesion of stomach; and C16.9 Malignant neoplasm: Stomach, unspecified.

\section{Data sources and variables}

The study was based on the analysis of official secondary mortality data. Mortality data were obtained from death certificates, as available from the nationwide Mortality Information System (Sistema de Informação sobre MortalidadeSIM) of the Brazilian Ministry of Health (MoH) (http://tabne t.datasus.gov.br/cgi/sim/dados/cid10_indice.htm). Brazilian physicians fill out standardized SIM forms, defining underlying and associated causes of death. The health secretaries of the municipalities are responsible for data entry, using a software package provided by the $\mathrm{MoH}$.

We downloaded and processed a total of 432 mortality data sets (one for each of the 27 Federal Units per year). Downloading and processing of these roughly 17 million records with mortality data from 2000 to 2015 was performed, as described previously [13].

Variables available and used for the analysis included: sex (male, female), age (grouped as $<15,15-29,30-39,40-49$, $50-59,60-69, \geq 70$ years; and $<45, \geq 45$ years), education, ethnicity (Caucasian, Afro-Brazilian, Asian, Mixed/PardoBrazilian and Amerindian), marital status, date of death, place of residence, place of occurrence of death, and underlying and associated causes of death.

Census data were obtained from the Brazilian Institute of Geography and Statistics (Instituto Brasileiro de Geografia e Estatística-IBGE, http://tabnet.datasus.gov.br/cgi/defto $\mathrm{htm}$.exe?ibge/cnv/popuf.def). We used information available from the national demographic census performed in 2000 and 2010, and inter census estimates for 2001-2009 and 2011-2015.

\section{Statistical analysis}

Age- and sex-adjusted mortality rates with their 95\% confidence intervals (CIs) were calculated by the direct method, with the 2010 Brazilian Census population as standard population, and expressed per 100,000 inhabitants. We excluded all records with incomplete data for any variables.

To compare GC-related mortality rates by population subgroups (sex, age group, region of residence, and ethnicity), we calculated the stratified crude and adjusted mortality rates, the rate ratios (RR) and the respective $95 \%$ CIs by means of bivariate analysis. The differences among groups were assessed by Pearson's Chi-squared test.

\section{Spatial analyses}

We used all 5570 Brazilian municipalities of residence as units of analysis (territorial division of 2013). Data records with unknown municipality of residence were excluded. The spatial distribution patterns of GC-related mortality were analyzed in four periods: 2000-2003, 2004-2007, 2008-2011, and 2012-2015. Average annual crude (unsmoothed) mortality rates (per 100,000 inhabitants) were calculated in each municipality for each time period, and over the entire study period (2000-2015), considering the population size in the middle of study period.

To correct for random variations and to provide a better stability of mortality rates particularly in small municipalities, we calculated smoothed mortality rates by the local empirical Bayesian smoothing method. This method adjusts the rates by incorporating data from neighboring municipalities with contiguous boundaries, and thus also reduced variation due to possible underreporting of deaths [14].

To identify significant spatial hot spots, cold spots and outliers of GC-related mortality rates, we evaluated local autocorrelation (local index of spatial association-LISA) by means of Local Moran's I index [15]. For spatial representation of the local Moran's index, Moran maps were drawn, considering municipalities with local spatial autocorrelation as significant ( $p$ value $<0.05$ ).

To verify the local spatial dependence from the mean rates of GC-related mortality rates, Gi* indexes (Gi star) of Getis-Ord were calculated. The result of the index ( $Z$ 
score and $p$ value) indicates significant spatial clustering, given the spatial relationships and scale of analysis (distance parameter). A high value of the $Z$ score and a small $p$ value indicate a spatial cluster of high values, whereas a low negative $Z$ score and a small $p$ value indicate a spatial clustering of low values.

Data analysis was performed using STATA software version 11.2 (StataCorp LP, College Station, TX, USA). Empirical Bayes smoothing estimates of GC-mortality rates were performed using the "Bayes Empirico Local" module of TerraView software version 5.3.1 (Instituto Nacional de Pesquisas Espaciais-INPE, São José dos Campos, SP, Brazil; available free at: http://www.dpi.inpe.br/terralib5/wiki/doku. php). Mapping was done with ArcGIS software package, version 9.3 (Environmental Systems Research Institute, Inc., Redlands, CA, USA).

\section{Ethics}

This study was approved in 2015 by the Ethical Review Board of the Federal University of Ceará (Fortaleza, Brazil), registration number 50537415.3.0000.5054, for gastric cancer mortality data from 2000 to 2013. In 2016, a new Resolution of the National Health Council was published, stating that there is no need to seek approval from any Ethical Review Board for studies using publicly available secondary data without identification of individual information (http://conselho.saude.gov.br/resolucoes/2016/reso510.pdf). In 2017, data were available on gastric cancer mortality up to 2015, and the scope of the analysis was amended for these additional 2 years. We subsequently contacted the Ethical Review Board, which clarified that a new submission was not necessary, considering the Resolution.

\section{Results}

Between 2000 and 2015, a total of 17,374,134 deaths were recorded. GC was identified in 214,808 deaths (1.24\%; $95 \%$ CI 1.23-1.24\%), 203,941 (94.9\%) as underlying cause, and $10,867(5.1 \%)$ as associated cause. The average annual number of GC-related deaths in the 16-year period was 13,426, ranging from 11,478 in 2000 to 15,080 in 2015.

The median age at GC-related death was 67.2 years, ranging from 30.7 to 93.9 years. Males [64.9\% $(139,330 / 214$, $808)], \geq 70$ year-olds $[45.6 \%(97,859 / 214,808)]$, Caucasians $[56.3 \%(120,955 / 214,808)]$, and residents in the Southeast region $[50.2 \%(107,893 / 214,808)]$ were the subgroups with the highest rates (Table 1).

The average annual adjusted rates for age and sex was 6.85 deaths/100,000 inhabitants (95\% CI 6.73-6.97), with a crude rate of 6.87 deaths $/ 100,000$ inhabitants. Average annual adjusted rates for age and sex were significantly higher in males than females. Age-specific rates increased with age, with 75.39 deaths/100,000 inhabitants older or equal to 70 years of age (95\% CI 73.48-77.30). The rates were 3.79 times higher in the group $\geq 45$ years of age than in those younger than 45 years of age (Table 1). Adjusted rates for age and sex (per 100,000 inhabitants) (95\% CI), from GC, Brazil and other regions, 2000-2015 are outlined in Table 2.

\section{Spatial distribution of mortality rates}

A total of 5435/5570 (97.6\%) Brazilian municipalities in all federal units registered GC-related death. Figures 1 and 2 present the spatial distribution of crude and smoothed GCrelated mortality rates. Average annual adjusted rates for age and sex rates reached a maximum of 6.85 deaths $/ 100,000$ inhabitants. The South (7.56 deaths/100,000 inhabitants) and the Southeast ( 7.36 deaths $/ 100,000$ inhabitants) regions had the highest regional mortality rates. In the 2000-2003 and 2004-2007 periods, we observed in more developed areas high crude and smoothed rates, mainly in the South and Southeast regions of Brazil (Figs. 2, 3). In these two time periods, the highest mortality rates of the Northeast, North and Central-West Brazilian regions were observed in the state of Ceará. In the most recent periods (2008-2011 and 2012-2015), other areas of the Northeast (Rio Grande do Norte, Paraíba e Pernambuco) and North (Pará) regions also had high mortality rates.

\section{Spatial cluster analysis}

For all GC-related deaths, global Moran's I index showed significant positive spatial autocorrelation in the four time periods: 2000-2003 (0.224; $p<0.001), 2004-2007$ (0.224; $p<0.001), 2008-2011(0.065 ; p<0.001)$, and 2012-2015 $(0.059 ; p<0.001)$, evidencing the existence of spatial dependence among GC-related rates. Figure 3 presents the clusters of municipalities identified according to LISA analysis for the mortality rates in the four time periods. During the first two periods, 2000-2007, we identified highrisk mortality clusters in South (Rio Grande do Sul, Santa Catarina and Paraná), Southeast (São Paulo, Rio de Janeiro, Minas Gerais and Espírito Santo), and Central-West (Mato Grosso and Mato Grosso do Sul) regions. In the two subsequent periods, 2008-2015, we observed high-risk areas in Northeast (Ceará, Rio Grande do Norte and Paraíba) and in North (Pará, Amazonas and Acre) regions. There was a large concentric cluster in South-Southeast Brazil, covering most municipalities of the states of Santa Catarina, Paraná, and São Paulo, extending to other neighboring states. We identified, in the last period, a smaller high-risk cluster located in the states of Pará, Amazonas, and Acre (Fig. 4). 
Table 1 Number of deaths, adjusted rates for age and sex (per 100,000 inhabitants), RR (95\% CI), $p$ value, from GC, stratified by sex, age groups, region of residence, and ethnicity, Brazil, 2000-2015

\begin{tabular}{|c|c|c|c|c|c|}
\hline Variables & $\begin{array}{l}\text { Deaths } \\
n(\%)\end{array}$ & $\begin{array}{l}\text { Crude mortality } \\
\text { Rate }(95 \% \text { CI) }\end{array}$ & $\begin{array}{l}\text { Adjusted rates for age and sex } \\
\text { Rate }(95 \% \mathrm{CI})\end{array}$ & $\mathrm{RR}(95 \% \mathrm{CI})$ & $p$ value \\
\hline All GC-related deaths & $214,808(100.0)$ & $6.87(6.75-6.99)$ & $6.85(6.73-6.97)$ & - & - \\
\hline \multicolumn{6}{|l|}{$\operatorname{Sex}^{b}$} \\
\hline Male & $139,330(64.9)$ & $9.00(8.82-9.2)$ & $10.00(9.79-10.21)$ & $1.8474(1.7835-1.9136)$ & $<0.0001$ \\
\hline Female & $75,458(35.1)$ & $4.87(4.74-5.01)$ & $4.34(4.21-4.46)$ & Ref & - \\
\hline \multicolumn{6}{|l|}{ Age group 1 (years) ${ }^{b}$} \\
\hline$<15$ & $56(0.0)$ & $0.01(0-0.02)$ & $0.01(0-0.01)$ & $0.0007(0.0002-0.0017)$ & $<0.0001$ \\
\hline $15-29$ & $1748(0.8)$ & $0.21(0.17-0.25)$ & $0.21(0.17-0.25)$ & $0.0171(0.0141-0.0207)$ & $<0.0001$ \\
\hline $30-39$ & $6617(3.1)$ & $1.37(1.25-1.51)$ & $1.37(1.23-1.5)$ & $0.1116(0.1005-0.1239)$ & $<0.0001$ \\
\hline $40-49$ & $18,417(8.6)$ & $4.54(4.28-4.81)$ & $4.59(4.33-4.86)$ & $0.3688(0.3435-0.396)$ & $<0.0001$ \\
\hline $50-59$ & $36,298(16.9)$ & $12.3(11.8-12.82)$ & $12.43(11.91-12.94)$ & Ref & - \\
\hline $60-69$ & $53,813(25.1)$ & $30.41(29.39-31.46)$ & $31.60(30.53-32.67)$ & $2.4717(2.3434-2.6071)$ & $<0.0001$ \\
\hline$\geq 70$ & $97,859(45.6)$ & $70.86(69.1-72.65)$ & $75.39(73.48-77.30)$ & $5.7572(5.4864-6.0413)$ & $<0.0001$ \\
\hline \multicolumn{6}{|l|}{ Age group 2 (years) $^{\mathrm{b}}$} \\
\hline$<45$ & $15,458(7.2)$ & $0.66(0.62-0.71)$ & $0.69(0.65-0.73)$ & Ref & - \\
\hline$\geq 45$ & $199,350(92.8)$ & $25.17(24.74-25.62)$ & $24.98(24.55-25.42)$ & $3.7891(3.5491-4.0453)$ & $<0.0001$ \\
\hline \multicolumn{6}{|l|}{ Region of residence ${ }^{b}$} \\
\hline North & $13,363(6.2)$ & $5.15(4.81-5.51)$ & $7.74(7.2-8.27)$ & Ref & - \\
\hline Northeast & $43,131(20.1)$ & $5.33(5.14-5.53)$ & $5.45(5.24-5.65)$ & $1.0348(0.9581-1.1176)$ & 0.3839 \\
\hline Southeast & $107,893(50.2)$ & $8.18(7.99-8.38)$ & $7.36(7.18-7.53)$ & $1.5884(1.4782-1.7068)$ & $<0.0001$ \\
\hline South & $38,621(18.0)$ & $8.36(8.03-8.71)$ & $7.56(7.25-7.86)$ & $1.6225(1.4993-1.7558)$ & $<0.0001$ \\
\hline Central-West & $11,800(5.5)$ & $5.16(4.8-5.55)$ & $5.92(5.48-6.35)$ & $1.0022(0.9077-1.1065)$ & 0.9660 \\
\hline \multicolumn{6}{|l|}{ Ethnicity $^{\mathrm{b}}$} \\
\hline Caucasian & $120,955(56.3)$ & $8.3(8.12-8.49)$ & $7.26(7.09-7.42)$ & Ref & - \\
\hline Afro-Brazilian & $16,290(7.6)$ & $7.01(6.59-7.46)$ & $6.69(6.28-7.1)$ & $0.8445(0.791-0.9016)$ & $<0.0001$ \\
\hline Asian & $2561(1.2)$ & $7.68(6.58-8.96)$ & $6.27(5.29-7.24)$ & $0.9246(0.7906-1.0813)$ & 0.3261 \\
\hline Mixed/Pardo-Brazilian & $60,393(28.1)$ & $4.59(4.44-4.74)$ & $5.5(5.33-5.68)$ & $0.5526(0.5314-0.5746)$ & $<0.0001$ \\
\hline Amerindian & $327(0.2)$ & $2.45(1.58-3.78)$ & $3.11(1.71-4.51)$ & $0.2945(0.1899-0.4567)$ & $<0.0001$ \\
\hline \multicolumn{6}{|l|}{ Marital status ${ }^{\mathrm{b}}$} \\
\hline Single & $36,286(16.9)$ & $2.53(2.43-2.64)$ & $7.91(7.56-8.26)$ & Ref & - \\
\hline Married/stable union & $113,491(52.8)$ & $12.57(12.28-12.86)$ & $7.75(7.51-7.98)$ & $4.9677(4.7383-5.2083)$ & $<0.0001$ \\
\hline Widow & $43,189(20.1)$ & $33.47(32.23-34.76)$ & $9.21(8.33-10.08)$ & $13.2274(12.5092-13.9868)$ & $<0.0001$ \\
\hline Divorced/separated & $10,456(4.9)$ & $13.03(12.07-14.07)$ & $5.76(5.25-6.26)$ & $5.1500(4.721-5.6181)$ & $<0.0001$ \\
\hline \multicolumn{6}{|l|}{ Education level (years) ${ }^{b}$} \\
\hline None & $33,242(15.5)$ & - & - & - & - \\
\hline $1-3$ & $52,622(24.5)$ & - & - & - & - \\
\hline $4-7$ & $38,913(18.1)$ & - & - & - & - \\
\hline $8-11$ & $19,336(9)$ & - & - & - & - \\
\hline$\geq 12$ & $9846(4.6)$ & - & - & - & - \\
\hline
\end{tabular}

$C I$ confidence intervals, $R R$ rate ratio, - not calculated

${ }^{a}$ Average annual crude rate (per 100,000 inhabitants), calculated using the average number of GC-related deaths in the sixteen-period as a numerator and population size in the middle of the studied period as a denominator. Population data on ehtnicity was derived from the Brazilian National Censuses (2000 and 2010). The population size by ehtnicity for the middle of the period was based on the Continuous National Household Sample Survey (PNAD) estimates

${ }^{\mathrm{b}}$ Data not available in all cases (sex: 20, ethnicity: 14,282, marital status: 11,386 and education level : 60,849)

The hot spot analysis (Getis-Ord Gi*) of GC-related mortality rates shows significant spatial clustering in South, Southeast, and Central-West regions in all time periods: $2000-2003(<0.001 ; p<0.001), 2004-2007$ $(<0.001 ; p<0.001), 2008-2011(<0.001 ; p<0.001)$, and 2012-2015 (<0.001; $p<0.001)$ (Fig. 4). In the 2008-2011 
Table 2 Adjusted rates for age and sex (per 100,000 inhabitants) (95\% CI), from GC, Brazil and other regions, 2000-2015

\begin{tabular}{lllllll}
\hline Year & Brazil & North & Northeast & Southeast & South & Central-West \\
\hline 2000 & $6.59(6.47-6.71)$ & $6.10(5.59-6.61)$ & $3.57(3.40-3.75)$ & $7.94(7.75-8.14)$ & $8.27(7.93-8.61)$ & $6.32(5.82-6.82)$ \\
2001 & $6.61(6.49-6.73)$ & $6.32(5.81-6.84)$ & $3.91(3.74-4.09)$ & $7.91(7.71-8.10)$ & $7.98(7.64-8.31)$ & $5.65(5.19-6.12)$ \\
2002 & $6.72(6.60-6.84)$ & $6.18(5.67-6.69)$ & $4.10(3.92-4.29)$ & $7.84(7.64-8.03)$ & $8.31(7.97-8.65)$ & $6.29(5.80-6.78)$ \\
2003 & $6.79(6.67-6.91)$ & $6.45(5.93-6.96)$ & $4.24(4.06-4.43)$ & $7.92(7.73-8.12)$ & $8.26(7.93-8.60)$ & $5.90(5.43-6.36)$ \\
2004 & $6.93(6.81-7.05)$ & $6.73(6.21-7.26)$ & $4.29(4.11-4.48)$ & $7.94(7.75-8.13)$ & $8.55(8.21-8.88)$ & $6.84(6.34-7.34)$ \\
2005 & $7.09(6.97-7.21)$ & $6.58(6.09-7.08)$ & $5.08(4.87-5.28)$ & $8.05(7.86-8.24)$ & $8.36(8.03-8.69)$ & $5.94(5.49-6.38)$ \\
2006 & $7.08(6.96-7.21)$ & $7.74(7.18-8.29)$ & $5.64(5.43-5.85)$ & $7.63(7.44-7.81)$ & $7.89(7.57-8.21)$ & $6.45(5.97-6.92)$ \\
2007 & $7.21(7.09-7.33)$ & $8.86(8.26-9.45)$ & $5.94(5.72-6.15)$ & $7.67(7.49-7.85)$ & $7.69(7.38-8.00)$ & $6.49(6.02-6.96)$ \\
2008 & $7.04(6.92-7.15)$ & $7.95(7.40-8.50)$ & $5.95(5.74-6.17)$ & $7.48(7.30-7.66)$ & $7.56(7.26-7.87)$ & $5.94(5.50-6.38)$ \\
2009 & $7.02(6.90-7.14)$ & $8.70(8.12-9.27)$ & $5.85(5.63-6.06)$ & $7.39(7.21-7.57)$ & $7.55(7.25-7.85)$ & $6.27(5.82-6.72)$ \\
2010 & $7.22(7.10-7.34)$ & $7.98(7.43-8.52)$ & $6.38(6.16-6.60)$ & $7.53(7.35-7.70)$ & $7.71(7.40-8.01)$ & $6.44(5.99-6.89)$ \\
2011 & $7.12(7.00-7.24)$ & $8.79(8.22-9.36)$ & $6.30(6.08-6.53)$ & $7.32(7.15-7.50)$ & $7.55(7.25-7.85)$ & $6.17(5.73-6.60)$ \\
2012 & $7.28(7.16-7.40)$ & $9.31(8.73-9.89)$ & $6.42(6.20-6.65)$ & $7.42(7.24-7.59)$ & $7.95(7.65-8.26)$ & $6.01(5.59-6.44)$ \\
2013 & $7.41(7.29-7.53)$ & $9.72(9.13-10.31)$ & $6.61(6.38-6.83)$ & $7.61(7.44-7.79)$ & $7.62(7.32-7.92)$ & $6.42(5.98-6.86)$ \\
2014 & $7.33(7.22-7.45)$ & $10.20(9.60-10.8)$ & $6.74(6.51-6.97)$ & $7.36(7.19-7.53)$ & $7.45(7.16-7.75)$ & $6.44(6.01-6.87)$ \\
2015 & $7.37(7.25-7.49)$ & $10.09(9.5-10.68)$ & $6.99(6.76-7.23)$ & $7.28(7.11-7.45)$ & $7.58(7.29-7.88)$ & $6.53(6.09-6.96)$ \\
\hline
\end{tabular}

Rate adjusted for age and sex $(95 \% \mathrm{CI})$
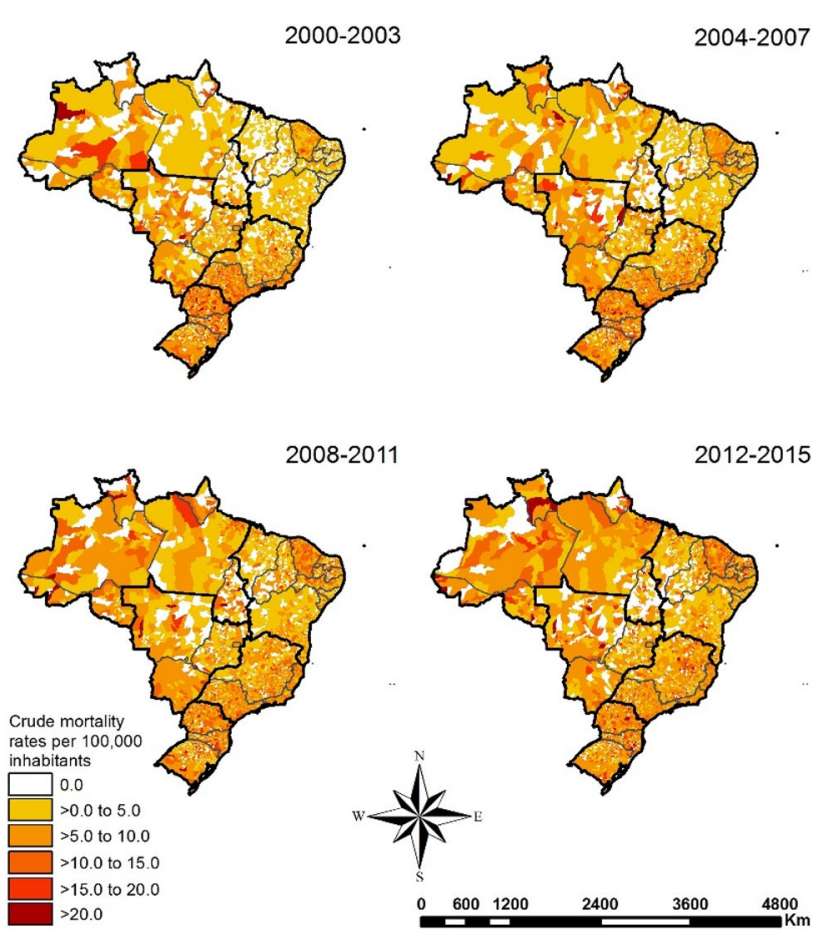

Fig. 2 Spatial distribution of average annual crude (non-smoothed) GC-related mortality rates (per 100,000 inhabitants) by municipality of residence in Brazil, 2000-2015

and 2012-2015 periods, Northeast (Ceará, Rio Grande do Norte and Paraíba) North (Pará, Amazonas and Acre) regions arise as high-risk areas (Fig. 5).
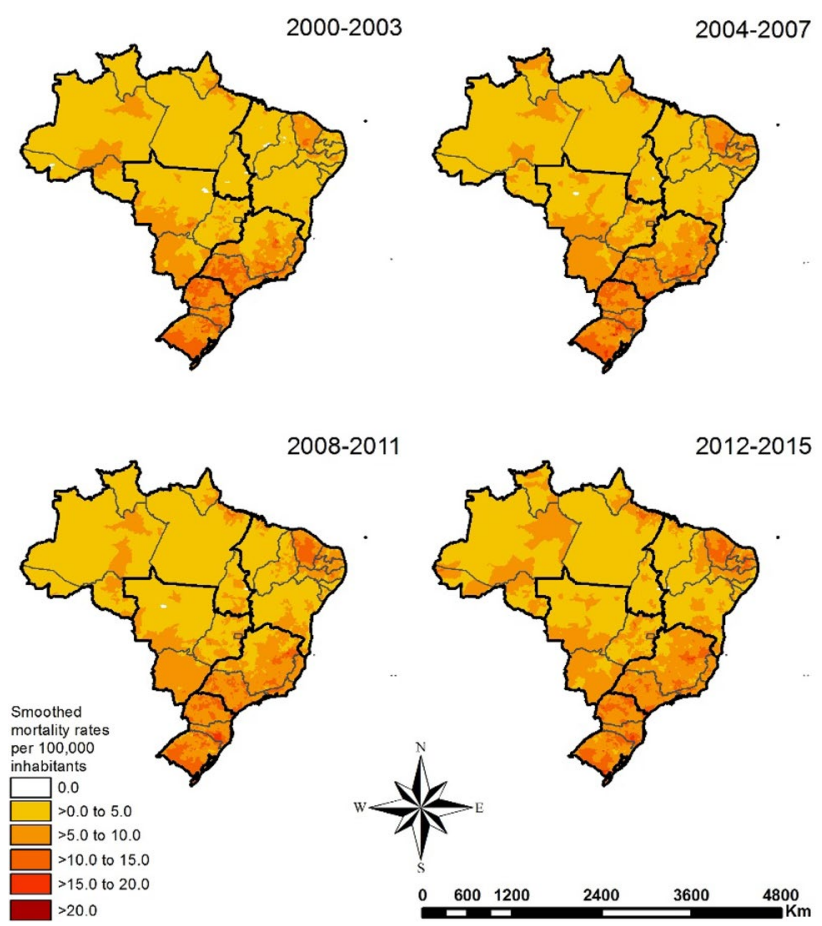

Fig. 3 Spatial distribution of average annual Bayesian-smoothed mortality rates (per 100,000 inhabitants) by municipality of residence in Brazil, 2000-2015

\section{Discussion}

Our systematic nationwide study is the first to describe the epidemiology of GC mortality in Brazil, using a 

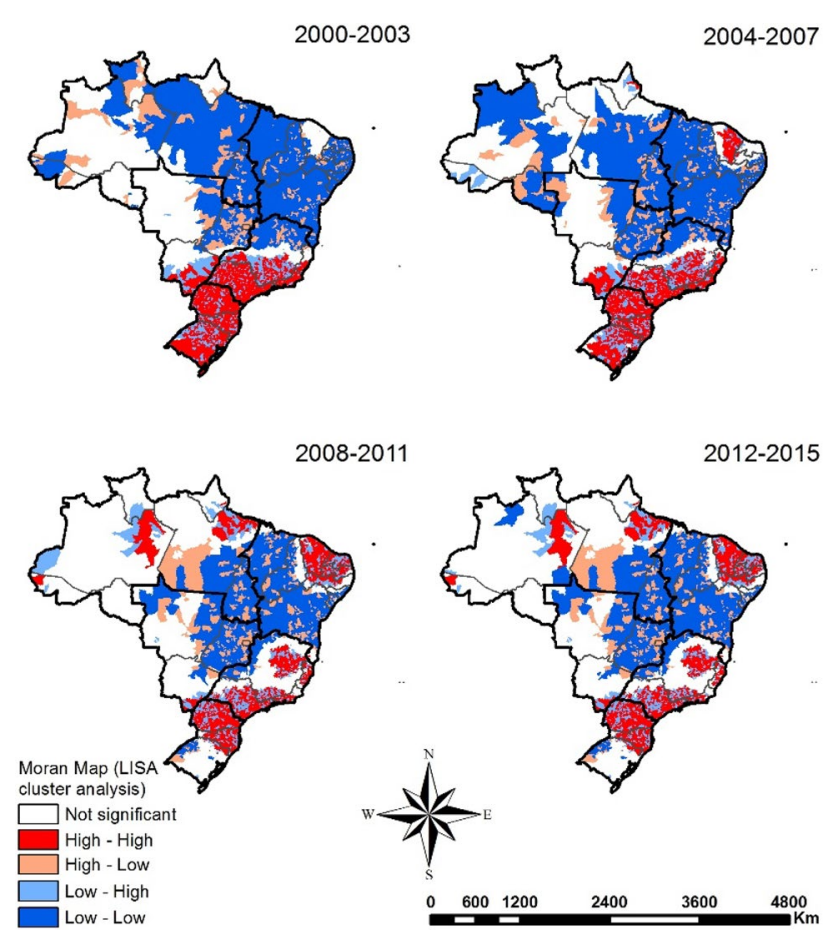

Fig. 4 Spatial and spatiotemporal LISA cluster analysis (Moran map) of GC-related mortality rates (per 100,000 inhabitants) by municipality of residence in Brazil, 2000-2015
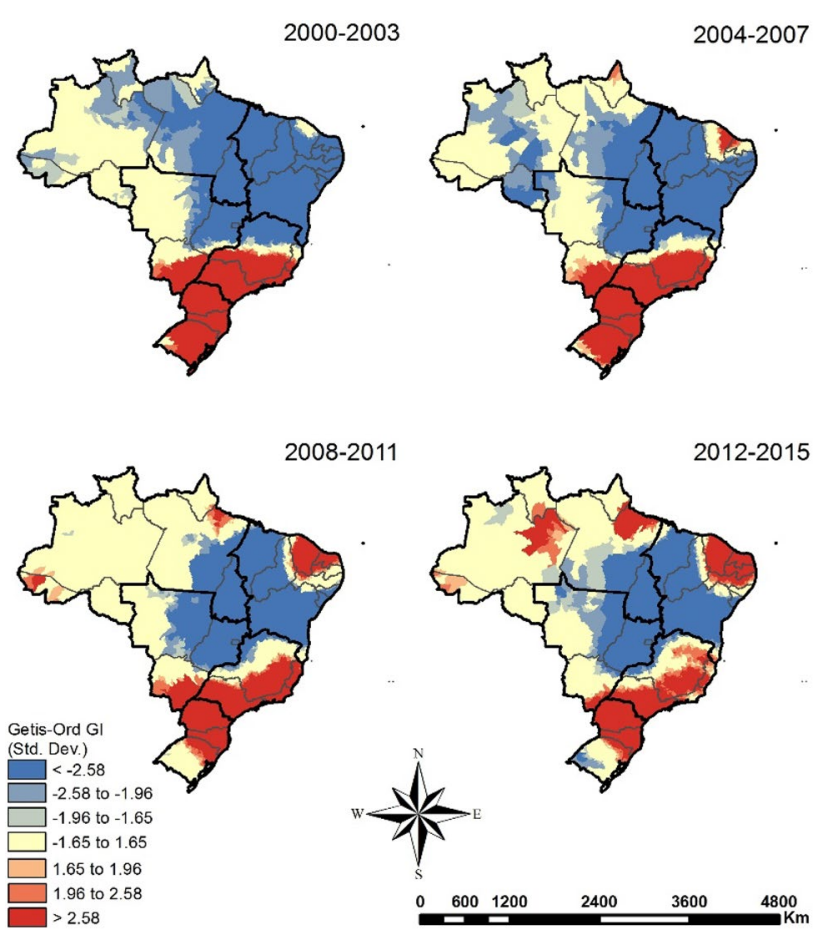

Fig. 5 Spatial and spatiotemporal hot spot analysis (Getis-Ord Gi*) of GC-related mortality rates (per 100,000 inhabitants) by municipality of residence in Brazil, 2000-2015 spatiotemporal approach. We found different mortality patterns across geographic regions and identified new hot spots and high-risk clusters for GC in the less developed regions (Northeast and North), in particular, in the State of Ceará. These findings highlight health inequalities and indicate the need to enhance an integrated network of health services, with special emphasis on less developed regions and vulnerable populations. The nationwide analysis spans a prolonged time period in a country of continental dimensions. Thus, our findings may hold true not only for Brazil, but may be generalized for other GC-affected countries worldwide.

The incidence of GC has decreased in most countries, likely due to declining prevalence of $H$. pylori infection and tobacco smoking, as well as improvement of the sanitation and access to refrigeration, which consequently decreased the need for salt as preservative [1, 5]. In 2015, there were an estimated 1.3 (1.2-1.4) million new cases worldwide of GC, 819,000 (795,000-844,000) deaths, and 17.4 (16.9-18) million disability-adjusted life years (DALYs) with $98.0 \%$ caused by years of life lost (YLLs) and $2 \%$ by years lived with disability (YLDs) [1]. Interestingly, while most countries have reported an increase of 5-year survival rates, in Brazil decreasing rates were observed [6, 9, 11, 12]. A previous study from Brazil has shown that the rates decreased in the Central-West, Southeast, and South regions, remained relatively stable in the North, and increased in the Northeast from 1980 to 2009 [11]. Another study has shown that the rates for 2005-2010 were higher in the South and Southeast regions; however, as the rates reduced in the Central-West and South, they proportionally increased in the Northern regions [12]. In our study, GC mortality rate was evaluated by including both underlying and associated causes of death, which increased GC mortality rate by about $5 \%$.

To identify hot spots and high-risk GC by municipality, we used a spatial-temporal analysis. We found that most of the municipality clusters of the South and Southeast regions of Brazil had higher GC mortality rates. Within the Northeast, North, and Central-West regions of Brazil, the state of Ceará is one of the most developed areas.

The analyses of the spatiotemporal clusters of GC-related mortality rates by municipality of residence showed an ongoing dynamic change within the country with a remarkable regional variations. During the period of 1999-2006, an important consistent change in the spatial cluster of municipalities in South, Southeast, and Central-West regions was observed. In the years thereafter, the least developed regions of the country, Northeast (Ceará, Rio Grande do Norte and Paraíba) and North region (Pará, Amazonas and Acre), became high-risk clusters for GC-related deaths with new GC municipality clusters emerging in rural areas. In contrast, during this period, mortality rates decreased in South, 
Southeast, and Central-West regions with better socioeconomic status in agreement with previous study [12].

The reported different regional GC mortality rates in Brazil can be partially explained by unequal regional socioeconomic levels and unequal health-care systems. The South and South-East of Brazil are considered the most socially and economically developed areas of the country, while the North and Northeast have been traditionally less developed areas. However, in the past decades the latter areas have developed more rapidly $[8,12]$, which likely led to better access to health care and improvement of death registry data. However, despite the fact that the state of Ceara was found to have such an improvement, even when compared with other states within the North and Northeast areas, it only by itself may not justify the increased GC mortality rates that occurred in the last years. Alternatively, changes in known risk factors involved in gastric carcinogenesis may have occurred, such as changes in the diet patterns as well as in the $H$. pylori infection prevalence (including possibly infection with more virulent strains). Among the dietary changes, the socioeconomic improvement may have led to increased consumption of unhealthy food, rich in starch, meat and fat, all of them associated with the risk of GC [16]. Other factors include an increased high salt intake due to increased use of industrialized food. Of note, salt has been shown to potentiate the colonization and virulence of $H$. pylori, [17] contributing to a high degree of gastric lesions and GC.

Infection by $H$. pylori plays a major role in gastric carcinogenesis and is considered the strongest risk factor [2, 18]. Studies conducted in several countries have shown that there is a robust association between GC and the infection with $H$. pylori $[2,18]$. In Brazil, the prevalence of $H$. pylori infection among adults ranges from 60.0 to $85.0 \%[19,20]$, with $80.0 \%$ of asymptomatic individuals from low-income community infected [20] and dyspeptic patients [21] in the State of Ceará.

In Ceará, patients are usually infected with the most virulent cagA-positive $H$. pylori strains that are strongly associated with GC [22, 23]. However, there are geographical differences in different regions, such as Africa and South Asia, where the prevalence of $H$. pylori infection is high, but the GC incidence rates are low [3]. It has been hypothesized that the genetics of the host and environmental factors may determine the infection outcomes [2, 24].

The increasing clusters of GC-related mortality rates by municipality of residence in rural areas observed in this study confirm previous reports from Brazil, China and South Korea [9, 25, 26]. During the 16-year study period, the highest mortality rates were found in males and in the oldest group in agreement with previous studies from Brazil [11], as well as worldwide [1,27]. We also found that the Asian ethnic group had the highest mortality rate, similar to previous studies from the USA where the incidence of GC in Japanese Americans was three to six times higher than in Caucasian Americans [28].

The present study has several limitations, such as the inclusion of secondary mortality data that may be underreported, despite the progress achieved in terms of SIM coverage and quality of information about causes of death [13]. Furthermore, the proportion of deaths from ill-defined causes may be distributed unequally between regions, urban and rural areas, age groups, and socioeconomic strata [11, 13]. The ratio between reported and estimated deaths in Brazil varied considerably, from 55.2\% in Maranhão state in the Northeast region to $100.0 \%$ in some states of the South and Southeast regions in 2000. The coverage improved steadily in 2011, when the regional differences became smaller, with the lowest coverage of $79.1 \%$ in Maranhão state, which may be due to increased coverage and improvement of death records of the SIM, as well as to the improvement of the access to health-care services. The use of information based on multiple causes of death (i.e., the mention of the same GC as cause of death in any part of the death certificate rather than only as the underlying cause) could reduce error. The focus on multiple cause of death of GC may result in an apparent artificial increase in statistical power and possibly misleading inferences. However, considering the nature of the study, the increase in sensitivity for the identification of CG deaths is justified.

Another limitation includes lack of information either in the proximal or distal location of the tumor, which has distinct risk factors, distributions, and outcomes. Distal GC is more prevalent in developing countries and $H$. pylori infection is a major risk factor along with smoking and dietary pattern [1,2]. Otherwise, proximal GC predominates in developed countries, with obesity, gastroesophageal reflux, and Barrett esophagus being the most important risk factors [24]. The incidence of proximal GC has been increasing, particularly in developed countries [1,24].

Despite the limitations, the data can be considered as highly representative, since all death certificates during 2000-2015 (more than 17 million of deaths) were included in a country of continental dimensions.

In conclusion, GC continues to be an important cause of death and a public health problem in Brazil. There is a change of GC mortality patterns by geographic regions over a 16-year period. Further studies are warranted, in particular in the areas of highest mortality, to identify associated risk factors and to design effective intervention measures.

Acknowledgements JH and DMMQ are class 1 research fellows at National Council for Scientific and Technological Development/Conselho Nacional de Desenvolvimento Científico e Tecnológico (CNPq/ Brazil). AFF is a master fellow at $\mathrm{CNPq} /$ Brazil.

Author contributions LLBCB, ANR Jr, and JH conceived and designed the study. ANR Jr, AFF, and CHA contributed to data collection and 
analysis. LLBCB, ANR Jr, MBBN, and JH wrote the manuscript. DMMQ, AFF, and DCM revised the manuscript. All authors contributed to the interpretation of data and approved the final manuscript.

Funding None.

\section{Compliance with ethical standards}

Conflict of interest We declare that we have no conflicts of interest.

\section{References}

1. Global Burden of Disease Cancer Collaboration, Fitzmaurice C, Allen C, Barber RM, et al. Global, regional, and national cancer incidence, mortality, years of life lost, years lived with disability, and disability-adjusted life-years for 32 cancer groups, 1990 to 2015: a systematic analysis for the global burden of disease study. JAMA Oncol 2017;3:524-48.

2. De Martel C, Forman D, Plummer M. Gastric cancer: epidemiology and risk factors. Gastroenterol Clin N Am. 2013;42:219-40.

3. Graham DY, Lu H, Yamaoka Y. African. Asian or Indian enigma, the East Asian Helicobacter pylori: facts or medical myths. J Dig Dis. 2009; 10:77-84

4. McLean MH, El-Omar EM. Genetics of gastric cancer. Nat Rev Gastroenterol Hepatol. 2014;11:664-74.

5. Zabaleta J. Multifactorial etiology of gastric cancer. Methods Mol Biol. 2012;863:411-35.

6. Sierra MS, Cueva P, Bravo LE, Forman D. Stomach cancer burden in Central and South America. Cancer Epidemiol. 2016;44:62-73.

7. Nashimoto A, Akazawa K, Isobe Y, et al. Gastric cancer treated in 2002 in Japan: 2009 annual report of the JGCA nationwide registry. Gastric Cancer 2013;16:1-27.

8. Instituto Nacional de. Câncer José Alencar Gomes da Silva. Coordenação de Prevenção e Vigilância. Estimate/2018-Cancer Incidence in Brazil. Rio de Janeiro: INCA; 2017.p. 128. http:// www.inca.gov.br/estimativa/2018/estimativa-2018.pdf. Accessed 5 June 2018

9. de Souza Giusti ACB, de Oliveira Salvador PTC, Dos Santos J, et al. Trends and predictions for gastric cancer mortality in Brazil. World J Gastroenterol. 2016;22:6527-38.

10. Uthman OA, Jadidi E, Moradi T. Socioeconomic position and incidence of gastric cancer: a systematic review and meta-analysis. J Epidemiol Community Health. 2013;67:854-60.

11. Guimarães RM, Muzi CD. Trend of mortality rates for gastric cancer in Brazil and regions in the period of 30 years (1980-2009). Arq Gastroenterol. 2012;49:184-8.

12. Amorim CA, Moreira JP, Rial L, et al. Ecological study of gastric cancer in Brazil: geographic and time trend analysis. World $\mathbf{J}$ Gastroenterol. 2014;20:5036-44.

13. Martins-Melo FR, Alencar CH, Ramos AN Jr, Heukelbach J. Epidemiology of mortality related to Chagas' disease in Brazil, 1999-2007. PLoS Negl Trop Dis. 2012;6:e1508.
14. Assunção RM, Schmertmann CP, Potter JE, Cavenaghi SM. Empirical Bayes estimation of demographic schedules for small areas. Demography. 2005;42:537-58.

15. Anselin L. Local indicators of spatial association-LISA. Geogr Anal. 1995;27:93-115.

16. Bertuccio P, Rosato V, Andreano A, et al. Dietary patterns and gastric cancer risk: a systematic review and meta-analysis. Ann Oncol. 2013;24:1450-8.

17. Wang X-Q, Terry PD, Yan H. Review of salt consumption and stomach cancer risk: epidemiological and biological evidence. World J Gastroenterol. 2009;15:2204-13.

18. Plummer M, Franceschi S, Vignat J, Forman D, de Martel C. Global burden of gastric cancer attributable to Helicobacter pylori. Int J Cancer. 2015;136:487-90.

19. Zaterka S, Eisig JN, Chinzon D, Rothstein W. Factors related to Helicobacter pylori prevalence in an adult population in Brazil. Helicobacter. 2007;12:82-8.

20. Rodrigues MN, Queiroz DM, Rodrigues RT, Rocha AM, Braga Neto MB, Braga LL. Helicobacter pylori infection in adults from a poor urban community in Northeastern Brazil: demographic, lifestyle and environmental factors. Braz J Infect Dis. 2005;9:405-10.

21. Motta CR, Cunha MP, Queiroz DM, Cruz FW, Guerra EJ, Mota RM, Braga LL. Gastric precancerous lesions and Helicobacter pylori infection in relatives of gastric cancer patients from Northeastern Brazil. Digestion. 2008;78:3-8.

22. Cavalcante MQ, Silva CI, Braga-Neto MB, et al. Helicobacter pylori vacA and cagA genotypes in patients from northeastern Brazil with upper gastrointestinal diseases. Mem Inst Oswaldo Cruz. 2012;107:561-3.

23. Queiroz DMM, Silva CISM, Goncalves MHRB, et al. Higher frequency of cagA EPIYA-C Phosphorylation sites in $H$. pylori strains from first-degree relatives of gastric cancer patients. BMC Gastroenterol. 2012;12:107.

24. Ang TL, Fock KM. Clinical epidemiology of gastric cancer. Singap Med J. 2014;55:621-28.

25. Guo P, Huang ZL, Yu P, Li K. Trends in cancer mortality in China: an update. Ann Oncol. 2012;23:2755-62.

26. Lee WJ, Son M, Chun BC, Park ES, Lee HK, Coble J, Dosemeci M. Cancer mortality and farming in South Korea: an ecologic study. Cancer Causes Control. 2008;19:505-13.

27. Anderson WF, Camargo MC, Fraumeni JF, Correa P, Rosenberg PS, Rabkin CS. Age-specific trends in incidence of noncardia gastric cancer in US adults. JAMA. 2010;303:1723-8.

28. Kamineni A, Williams MA, Schwartz SM, Cook LS, Weiss NS. The incidence of gastric carcinoma in Asian migrants to the United States and their descendants. Cancer Causes Control. 1999;10:77-83.

Publisher's Note Springer Nature remains neutral with regard to jurisdictional claims in published maps and institutional affiliations. 